\section{INNATE IMMUNITY}

\section{C. elegans an innate choice?}

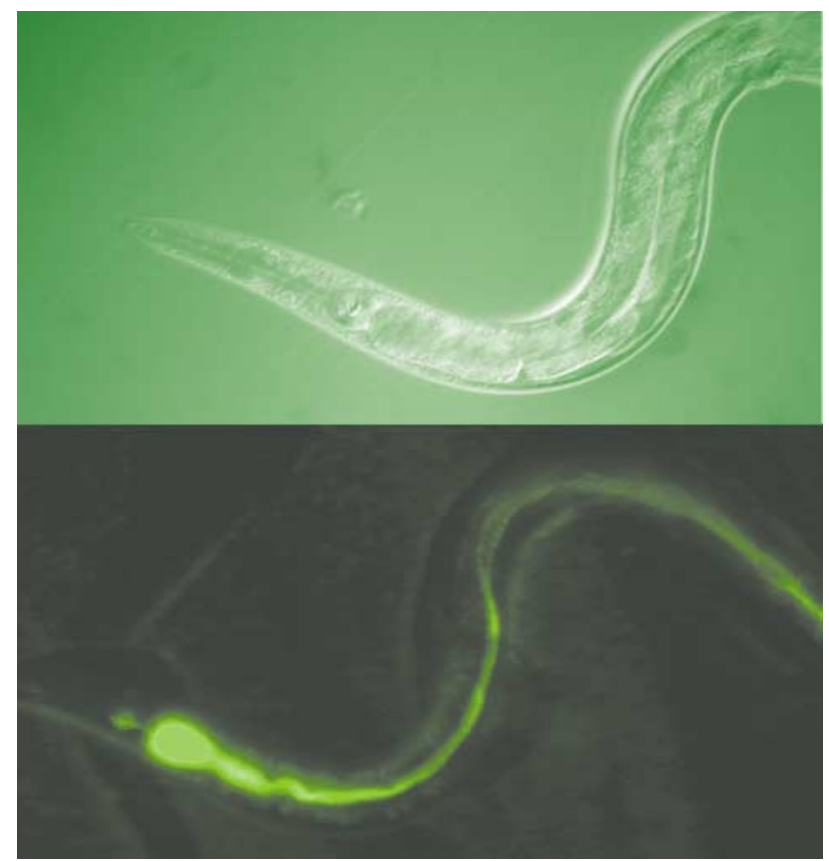

An esp2 mutant worm, after exposure to GFP-labelled $P$. aeruginosa. Nomarski (top) and fluorescence (bottom) images courtesy of Dennis Hyong-Kun Kim, Frederick Ausubel and fluorescence (bottom)
Much of what we know about innate immunity comes from studies in Drosophila.Two papers now show that Caenorhabditis elegans, another genetically tractable organism, might be a useful additional model for studying innate immunity. The results show that C. elegans has an inducible response to pathogen infection and that this response shares many features with innate immunity in other organisms.

Kim et al. assayed the progeny of mutagenized worms, which had been exposed to the bacterium Pseudomonas aeruginosa, for enhanced susceptibility to pathogen (esp) infection. Two mutants, esp2 and esp8, that had severe phenotypes were isolated both die much faster after exposure to $P$. aeruginosa than wild-type worms. The mutant genes were identified by phenotypic rescue - the esp 2 mutant was rescued by the gene sek-1, and esp 8 by $n s y-1$.

sek-1 encodes a mitogen-activated protein kinase kinase (MAPKK) homologue of mammalian MKK3/ MKK6 and MKK4, and nsy-1 encodes an orthologue of the mammalian MAPKKK ASK1. Because these kinases activate the p38 kinase family and the JNK MAPKs in mammals, the authors tested the role of p38 and JNK in the C. elegans defence response. The esp2 and esp 8 mutants had markedly reduced levels of p38 MAPK activity. Moreover, the knockdown of pmk-1, one of two C. elegans p38 orthologues, by RNA interference produced a strong esp phenotype. Knockdown of pmk-2 and a jnk mutation, however, produced no enhanced susceptiblity to $P$. aeruginosa infection. Together, these results show that the p38 MAPK pathway is required for innate responses to pathogen infection, which is an important discovery as this signalling pathway is also crucially required in mammals for inflammatory and innate-immune response signalling pathways.

Mallo et al. used an expression screen to look for C. elegans genes that are upregulated in response to infection by the bacterium Serratia marcescens. Of 7,500 cDNAs that were surveyed, several were induced more than twofold; most of these encode lectins, which function in innate immunity in other organisms. Lysozyme 1 was also upregulated. As lysozymes have been implicated in innate-immune responses, Mallo et al. overexpressed lys-1 in C. elegans to see if this would enhance resistance to
TUMOUR IMMUNITY

\section{Plugging the gap}

Most of the tumour antigens that have been identified are abberantly expressed selfmolecules that are poorly immunogenic This realization, together with the fact that normal individuals have $T$ cells that react against self-antigens, but are held in check by peripheral-tolerance mechanisms, has resulted in attempts to develop anti-tumour therapies that stimulate these anti-self $\mathrm{T}$ cells and elicit anti-tumour responses. A new study in The Journal of Clinical Investigation shows that stimulating the homeostatic proliferation of a polyclonal population of adoptively transferred $\mathrm{T}$ cells - including those that are specific for tumour selfantigens - in lymphopaenic animals can induce anti-tumour immunity.

Mice with lymphopaenia (induced by sublethal irradiation) and wild-type mice were challenged with a subcutaneous injection of melanoma cells, and tumour growth was assessed after 52 days. The lymphopaenic mice developed smaller tumours than wild-type mice. This anti-tumour effect was enhanced in a dose-dependent manner by the adoptive transfer of syngeneic lymph-node $T$ cells before challenge with the tumour cells. In vitro testing of the homeostatically expanded $\mathrm{T}$ cells showed that they specifically recognised the melanoma tumour cells, but not unrelated colon carcinoma cells.

Next, the authors investigated whether T-cell clonal expansion per se was sufficient to induce the anti-tumour response or whether antigen presentation of tumour antigens was also important. The inhibition of tumour growth was more efficient in wild-type lymphopaenic mice transfused with $\mathrm{T}$ cells and challenged with melanoma than in lymphotoxin- $\alpha$-deficient mice, which lack lymph nodes. When $\mathrm{T}$ cells that cannot traffic through lymph nodes (because they do not express $\beta 7$-integrin or L-selectin) were transferred, they were much less effective than normal $\mathrm{T}$ cells at eliciting the antitumour response. Together, these results show that the proliferating $\mathrm{T}$ cells need to be exposed to tumour antigens that are presented in the lymph nodes.
When mice that had rejected the melanoma cells were rechallenged with either melanoma or colon carcinoma cells, they again rejected the melanoma, but not the colon carcinoma, tumours, which indicates that the T-cell homeostatic proliferation had resulted in the development of specific long-term antitumour immunity.

Could homeostatic proliferation be useful for the treatment of established tumours? Lymphopaenic mice were challenged with melanoma cells, and when the tumours reached a specific size, the mice were transfused with lymph-node cells. Tumour growth was markedly inhibited in treated mice compared with controls.

These data indicate that, in the clinic, it might be beneficial to begin tumour immunotherapy soon after the completion of chemotherapy to induce effective homeostatic T-cell proliferation.

Elaine Bell

(4) References and links ORIGINAL RESEARCH PAPER Dummer, W. et al. T-cell homeostatic proliferation elicits effective antitumo autoimmunity. J. Clin. Invest. 110, 185-192 (2002) FURTHER READING Jameson, S. Maintaining the norm: T-cell homeostasis. Nature Rev. Immunol. 2, 547-556 (2002) 